\title{
Simulation Performance of WAVE System with Combined DD-CE and LMMSE Smoothing Scheme in Small-Scale Fading Models
}

\author{
Jeongwook Seo, Jae-Min Kwak and Dong Ku Kim, Member, KIMICS
}

\begin{abstract}
This paper investigates the performance of IEEE 802.11p wireless access in vehicular environments (WAVE) system in small-scale fading models reported by Georgia Institute of Technology (Georgia Tech). We redesign the small-scale fading models to be applied to the computer simulation and develop the IEEE 802.11p WAVE physical layer simulator to provide the bit error rate and packet error rate performances. Moreover, a new channel estimator using decision directed channel estimation and linear minimum mean square error smoothing is proposed in order to improve the performance of the conventional least square channel estimator using two identical long training symbols. The simulation results are satisfactorily coincident with the scenarios of Georgia Tech report, and the proposed channel estimator significantly outperforms the conventional channel estimator.
\end{abstract}

Index Terms-IEEE 802.11p, WAVE, channel modeling, channel estimation.

\section{INTRODUCTION}

IEEE $802.11 \mathrm{p}$ wireless access in vehicular environments (WAVE) is a dedicated short range communication (DSRC) radio technology. Its scope is to create an amendment of the overall IEEE 802.11 standard to support vehicle-to-vehicle (V2V) and roadside-tovehicle (R2V) communications [1]-[3]. The IEEE $802.11 \mathrm{p}$ standard describes the medium access control (MAC) and physical (PHY) layers. Especially, the PHY layer is similar to that of IEEE 802.11a wireless local area network (WLAN) since the design philosophy of the IEEE $802.11 \mathrm{p}$ is to make the minimum changes to the commercialized IEEE 802.11a to communicate among fast moving vehicles in the roadway environments [1]. By contrast with the IEEE 802.11a, the IEEE 802.11p exploits orthogonal frequency division multiplexing (OFDM) transmission with $10 \mathrm{MHz}$ channel spacing at $5.9 \mathrm{GHz}$ band. The reason to reduce the channel spacing is to deal with the increased root mean square (RMS) delay spread in vehicular environments. Moreover, the

Manuscript received April 14, 2010; revised May 3, 2010; accepted May 15, 2010.

Jeongwook Seo is with Advanced Mobile Technology Research Center, KETI, Seoul, 121-835, Korea (Email: jwseo@keti.rekr)
IEEE $802.11 \mathrm{p}$ introduces four spectrum masks for class A to $\mathrm{D}$ operations and some receiver performance requirements in adjacent channel rejections.

Wireless channel modeling is critical for the performance evaluation of the WAVE system [4]-[6]. Recently, Georgia Institute of Technology (Georgia Tech) announced the final technical report describing the channel models for WAVE operations [4], [5]. It presents six smallscale fading models where three of them are for the V2V link and the other three are for R2V link. Although a previous literature represented their channel models and evaluated the performance of the WAVE/DSRC system as shown in [6], they did not consider concrete channel environments. Therefore, we re-design the six small-scale fading models based on the Georgia Tech report to be proper for the computer simulation and evaluate the bit error rate (BER) and packet error rate (PER) performances of the WAVE PHY layer. The channel models and the simulation results can be used as performance references to implement and verify the WAVE system. In addition, we propose an improved channel estimator using decision directed channel estimation (DD-CE) and linear minimum mean square error (LMMSE) smoothing, since the conventional channel estimator using two identical long training symbols does not work well in the small-scale fading models.

This paper is organized as follows. Section II introduces IEEE $802.11 \mathrm{p}$ WAVE system model. Section III reviews small-scale fading channel models given by Georgia Tech. Section IV gives some simulation results to evaluate the performances of the IEEE 802.11p WAVE system and the proposed channel estimator. This paper is concluded in Section V.

\section{IEEE802.11P WAVE SYSTEM}

The WAVE system operating at 5.850 5.925 GHz band is described in this section. In Fig. 1, the WAVE PHY system model is shown. In the transmitter part, the short and long training symbols are generated and transmitted at first. Then arbitrary data bits are randomly generated and encoded by convolutional encoder with $1 / 2$ code rate. Then the encoded bits are punctured to support various data rates. The interleaver is exploited to change burst errors into random errors. The interleaved bits are modulated by BPSK, QPSK, 16QAM, or 64QAM. The 48 modulated symbols, the 4 pilot symbols, and the 12 null 
symbols are inserted into 64-point inverse fast Fourier transform (IFFT). After that, 16 cyclic prefix (CP) samples are added to the 64-point output samples of the IFFT operation to make an OFDM symbol. The CP samples are guard interval (GI) samples to prevent intersymbol interference (ISI) and inter-carrier interference (ICI). In order to suppress the out-of-band spectrum, the OFDM symbol is multiplied by a raised-cosine window.

In the receiver part, assuming perfect time and frequency synchronization, the GI samples are removed before the FFT operation. In order to compensate the channel distortion, one-tap equalizer is used for the received data symbols. The demodulation is performed for the equalized data symbols to produce the demodulated data bits, followed by deinterleaving and depuncturing operations. Viterbi algorithm using softdecision decoding and channel frequency response in [7] is used to produce the sink bits which shall be compared with the transmitted bits in order to calculate the BER and PER. The WAVE system parameters are shown in Table I. The subcarrier frequency spacing is $0.15625 \mathrm{MHz}$ given by the division $10 \mathrm{MHz} / 64$ subcarriers. The IFFT/FFT period is 6.4 us given by a reciprocal of the subcarrier frequency spacing. The GI duration is 1.6 us, the OFDM symbol duration is 8.0 us, and the PLCP preamble duration is 32 us.

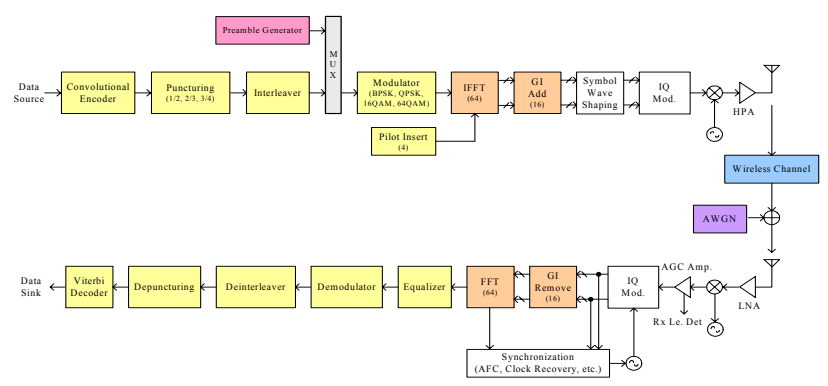

Fig. 1. WAVE physical layer system model.

The WAVE transmission modes are given in Table II. The maximum data rate is $27 \mathrm{Mbps}$ assuming 3/4 code rate and 64QAM modulation, and the minimum data rate is 3 Mbps assuming 1/2 code rate and BPSK modulation. Each data rate in Table II is half the data rate given in IEEE 802.11a.

TABLE I

WAVE SYSTEM PARAMETERS

\begin{tabular}{|l|l|}
\hline \multicolumn{1}{|c|}{ Parameters } & \multicolumn{1}{c|}{$\begin{array}{c}\text { Value } \\
(10 \mathrm{MHz} \text { channel } \\
\text { spacing })\end{array}$} \\
\hline \hline $\mathrm{N}_{\mathrm{SD}}:$ Number of data subcarriers & 48 \\
\hline $\mathrm{N}_{\mathrm{SP}}:$ Number of pilot subcarriers & 4 \\
\hline $\mathrm{N}_{\mathrm{ST}}:$ Number of subcarriers, total & $52\left(\mathrm{~N}_{\mathrm{SD}}+\mathrm{N}_{\mathrm{SP}}\right)$ \\
\hline$\Delta_{\mathrm{F}}:$ Subcarrier frequency spacing & $0.15625 \mathrm{MHz}$ \\
$(10 \mathrm{MHz} / 64)$
\end{tabular}

\begin{tabular}{|l|l|}
\hline $\mathrm{T}_{\mathrm{FFT}}$ : IFFT/FFT period & $6.4 \mu \mathrm{s}\left(1 / \Delta_{\mathrm{F}}\right)$ \\
\hline $\mathrm{T}_{\mathrm{PREAMBLE}}$ : PLCP preamble duration & $\begin{array}{l}32 \mu \mathrm{s} \\
\left(\mathrm{T}_{\mathrm{SHORT}}+\mathrm{T}_{\mathrm{LONG}}\right)\end{array}$ \\
\hline $\begin{array}{l}\mathrm{T}_{\mathrm{SIGNAL}} \text { : Duration of the SIGNAL BPSK- } \\
\text { OFDM symbol }\end{array}$ & $8.0 \mu \mathrm{s}\left(\mathrm{T}_{\mathrm{GI}}+\mathrm{T}_{\mathrm{FFT}}\right)$ \\
\hline $\mathrm{T}_{\mathrm{GI}}$ : GI (Guard Interval) duration & $1.6 \mu \mathrm{s}\left(\mathrm{T}_{\mathrm{FFT}} / 4\right)$ \\
\hline $\mathrm{T}_{\mathrm{GI} 2}$ : Training symbol GI duration & $3.2 \mu \mathrm{s}\left(\mathrm{T}_{\mathrm{FFT}} / 2\right)$ \\
\hline $\mathrm{T}_{\mathrm{SYM}}$ : Symbol interval & $8.0 \mu \mathrm{s}\left(\mathrm{T}_{\mathrm{GI}}+\mathrm{T}_{\mathrm{FFT}}\right)$ \\
\hline $\mathrm{T}_{\mathrm{SHORT}}$ : Short training sequence duration & $\begin{array}{l}16.0 \mu \mathrm{s} \\
\left(10 \times \mathrm{T}_{\mathrm{FFT}} / 4\right)\end{array}$ \\
\hline $\mathrm{T}_{\mathrm{LONG}}$ : Long training sequence duration & $\begin{array}{l}16.0 \mu \mathrm{s} \\
\left(\mathrm{T}_{\mathrm{GI}}+2 \times \mathrm{T}_{\mathrm{FFT}}\right)\end{array}$ \\
\hline
\end{tabular}

TABLE II

WAVE TRANSMISSION MODES

\begin{tabular}{|c|c|c|c|c|c|}
\hline Modulation & $\begin{array}{c}\text { Code } \\
\text { Rate }(\mathrm{R})\end{array}$ & $\begin{array}{c}\text { Coded bits } \\
\text { per } \\
\text { subcarrier } \\
\left(\mathrm{N}_{\text {BPSC }}\right.\end{array}$ & $\begin{array}{c}\text { Coded bits } \\
\text { per OFDM } \\
\text { symbol } \\
\left(\mathrm{N}_{\text {CBPC }}\right.\end{array}$ & $\begin{array}{c}\text { Data bits per } \\
\text { OFDM } \\
\text { symbol } \\
\left(\mathrm{N}_{\text {BpPS }}\right)\end{array}$ & $\begin{array}{c}\text { Data rate } \\
\text { (Mbps): } 10 \\
\text { MHz } \\
\text { channel } \\
\text { spacing }\end{array}$ \\
\hline \hline BPSK & $1 / 2$ & 1 & 48 & 24 & 3 \\
\hline BPSK & $3 / 4$ & 1 & 48 & 36 & 4.5 \\
\hline QPSK & $1 / 2$ & 2 & 96 & 48 & 6 \\
\hline QPSK & $3 / 4$ & 2 & 96 & 72 & 9 \\
\hline 16-QAM & $1 / 2$ & 4 & 192 & 96 & 12 \\
\hline 16-QAM & $3 / 4$ & 4 & 192 & 144 & 18 \\
\hline 64-QAM & $2 / 3$ & 6 & 288 & 192 & 24 \\
\hline 64-QAM & $3 / 4$ & 6 & 288 & 216 & 27 \\
\hline
\end{tabular}

\section{A. Proposed Channel Estimation}

We assume that the time and frequency synchronization is perfect, the multipath fading channel is quasi-stationary, and the $\mathrm{CP}$ length is longer than the maximum excess delay of the fading channel. The received symbol, after removing the $\mathrm{CP}$ and completing the FFT operation, is represented as

$$
Y_{l, k}=H_{l, k} X_{l, k}+W_{l, k}
$$
where $Y_{l, k}, H_{l, k}, X_{l, k}$, and $W_{l, k}$ are the received symbol, the channel frequency response, the transmitted symbol, and the noise term at the $k$ th subcarrier in the $l$ th OFDM symbol, respectively. The conventional least square (LS) channel estimator using two identical long training symbols in [7] is represented as follows

$$
\hat{H}_{k}=\frac{1}{2}\left(Y_{1, k}+Y_{2, k}\right) X_{k}^{*}=H_{k}+\frac{1}{2}\left(W_{1, k}+W_{2, k}\right) X_{l, k}^{*}
$$

where $X_{k}=X_{1, k}=X_{2, k}$ denotes the long training symbol. Moreover, $H_{k}=H_{1, k}=H_{2, k}$ is assumed. The same channel estimate $\hat{H}_{k}$ shall be used for the following OFDM symbols without updating themselves.

In order to improve the performance of the conventional LS channel estimator, we propose a new channel estimator combining DD-CE and LMMSE smoothing as shown in Fig. 2. 


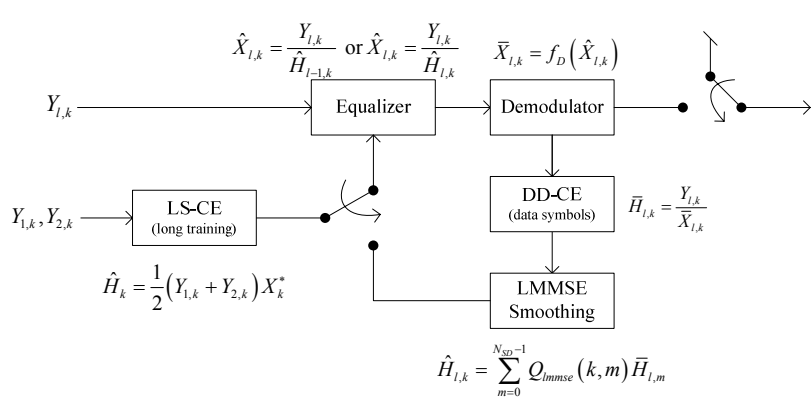

Fig. 2. The proposed channel estimator combining decision directed channel estimation (DD-CE) and linear minimum mean square error (LMMSE) smoothing.

The output of the one-tap equalizer is represented as

$\hat{X}_{l, k}=\frac{Y_{l, k}}{\hat{H}_{l-1, k}}=\frac{H_{l, k}}{\hat{H}_{l-1, k}} X_{l, k}+\frac{W_{l, k}}{\hat{H}_{l-1, k}} \quad$ for $\quad l \geq 3$

where $\hat{X}_{l, k}$ is the equalized symbol by the previous channel estimate $\hat{H}_{l-1, k}$. Then, the demodulation is performed via hard-decision function $f_{D}(\cdot)$ as shown below

$$
\bar{X}_{l, k}=f_{D}\left(\hat{X}_{l, k}\right)
$$

After that, the DD-CE and the LMMSE smoothing are represented as shown in (5) and (6)

$$
\begin{aligned}
\bar{H}_{l, k} & =\frac{Y_{l, k}}{\bar{X}_{l, k}} \\
\hat{H}_{l, k} & =\sum_{m=0}^{N_{S D}-1} Q_{l m m s e}(k, m) \bar{H}_{l, m}
\end{aligned}
$$

where $Q_{\text {lmmse }}(k, m)$ denotes the LMMSE smoothing coefficients, which is represented as

$$
Q_{\text {lmmse }}(k, m)=\left[\mathbf{R}_{\mathrm{H} \hat{\mathrm{H}}} \mathbf{R}_{\hat{\mathrm{H}} \hat{\mathrm{H}}}^{-1}\right]_{k, m}
$$

where $\mathbf{R}_{\mathrm{HH}}=E\left(\mathrm{HH}^{H}\right)$ is the cross-correlation matrix of the desired channel vector and the estimated channel vector, and $\mathbf{R}_{\hat{\mathrm{H} H}}=E\left(\hat{\mathrm{H}} \hat{\mathrm{H}}^{H}\right)$ is the autocorrelation matrix of the estimated channel vector. Here, $E(\cdot)$ denotes the expectation function, and $H$ denotes the Hermitian transpose. Note that the cross- and autocorrelation matrices can be pre-computed and stored when a uniform channel power delay profile in [8] is considered.

\section{SMALL-SCALE FADING MODELS}

This section describes six small-scale fading models based on the final technical report from Georgia Tech in [4]. These models denote typical WAVE environments for $\mathrm{V} 2 \mathrm{~V}$ and $\mathrm{R} 2 \mathrm{~V}$ links: 1) $\mathrm{V} 2 \mathrm{~V}$-expressway oncoming without wall, 2) R2V-urban canyon, 3) R2V-expressway, 4) V2V-urban canyon oncoming, 5) R2V-suburban street,
6) V2V-expressway same direction with wall as shown in Table III.

TABLE III

$$
\begin{aligned}
& \text { SMALL SCALE FADING CHANNEL MODELS BY } \\
& \text { GEORGIA TECH [4] }
\end{aligned}
$$

\begin{tabular}{|c|l|c|}
\hline Model & \multicolumn{1}{|c|}{ Scenario } & $\begin{array}{c}\text { PSDU } \\
\text { size } \\
{[\text { bytes] }}\end{array}$ \\
\hline \hline 1 & $\begin{array}{l}|c| \\
\text { V2V-Expressway Oncoming without Wall, } \\
300 \mathrm{~m}-400 \mathrm{~m} \text { Model }\end{array}$ & 200 \\
\hline 2 & R2V-Urban Canyon, 100 m Short Model & 1000 \\
\hline 3 & R2V-Urban Canyon, 100 m Long Model & 1000 \\
\hline 4 & R2V-Expressway, 300m-400m 140 km/hr Model & 200 \\
\hline 5 & R2V-Expressway, 300m-400m 200 km/hr Model & 200 \\
\hline 6 & $\begin{array}{l}\text { V2V-Urban Canyon Oncoming, 100m Short } \\
\text { Model }\end{array}$ & 1000 \\
\hline 7 & $\begin{array}{l}\text { V2V-Urban Canyon Oncoming, 100m Long } \\
\text { Model }\end{array}$ & 1000 \\
\hline 8 & R2V-Suburban Street, 100m Short Model & 1000 \\
\hline 9 & R2V-Suburban Street, 100m Long Model & 1000 \\
\hline 10 & $\begin{array}{l}\text { V2V-Expressway Same Direction with Wall, } \\
300 m-400 m \text { Model }\end{array}$ & 200 \\
\hline
\end{tabular}

The R2V-urban canyon, the V2V-urban canyon oncoming, and the $\mathrm{R} 2 \mathrm{~V}$ - suburban street have both long and short versions which are greater than 12 paths and less than or equal to 12 paths, respectively. All models are based on the measured data in spring 2006 at $5.9 \mathrm{GHz}$ in the metropolitan Atlanta, Georgia area and created by the fitting work with a standard RF channel emulator such as the SPIRENT SR5500. The vehicle speeds during measurement were approximately $105 \mathrm{~km} / \mathrm{hr}$ for the expressway and $32 \mathrm{~km} / \mathrm{hr}$ to $48 \mathrm{~km} / \mathrm{hr}$ for the surface streets. For the models in the report, the Doppler frequencies were scaled to be consistent with vehicle speeds of $140 \mathrm{~km} / \mathrm{hr}$ and $120 \mathrm{~km} / \mathrm{hr}$, respectively.

Each model is of the tapped delay line (TDL) type and normalized for the first tap power to be $0 \mathrm{~dB}$. In most cases, a tap is constructed from several paths of the channel emulator because the choices for the spectral shapes of paths are limited and quite insufficient to describe many of the measured tap Doppler spectra. By superimposing several paths with nearly the same delay, it is possible to create a customized Doppler spectrum fit better. The customized Doppler spectrum has the same total power as the measured Doppler spectrum for the power delay profile to be preserved. Moreover, a genetic algorithm was used to optimize each tap spectrum fit.

We designed the channel models to be applied to the computer simulation for the performance evaluation of the WAVE system in MATLAB environment. As an example of our work, the last model of V2V-expressway same direction with wall $300 \mathrm{~m}-400 \mathrm{~m}$ in Table III is considered. In Fig. 3, the measurement environment is shown where the transmitter vehicle keeps a $400 \mathrm{~m}$ separation to the front of the receiver vehicle and there is a center wall between oncoming lanes. 


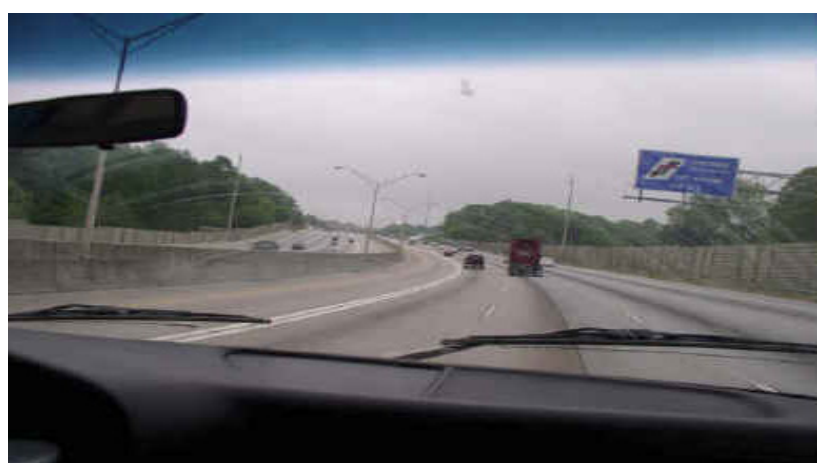

Fig. 3. Typical conditions for V2V-expressway same direction with wall.

\section{TABLE IV}

V2V EXPRESSWAY SAME DIRECTION WITH WALL (Note: (1) Tap no., (2) Path num., (3) Tap power (dB), (4) Relative path loss (dB), (5) Delay value (ns), (6) Rician K (dB), (7) Freq. shift (Hz), (8) Fading Doppler (Hz), (9) LOS Doppler (Hz), (10) Modulation, (11) Fad. spec. shape.)

\begin{tabular}{|c|c|c|c|c|c|c|c|c|c|c|}
\hline$(1)$ & $(2)$ & $(3)$ & $(4)$ & $(5)$ & $(6)$ & $(7)$ & $(8)$ & $(9)$ & $(10)$ & $(11)$ \\
\hline \hline 1 & 1 & 0.0 & -1.4 & 0 & 23.8 & -55 & 1407 & -60 & Rician & Round \\
\hline 1 & 2 & & -5.6 & 1 & $\mathrm{n} / \mathrm{a}$ & -20 & 84 & $\mathrm{n} / \mathrm{a}$ & Rayleigh & Round \\
\hline 2 & 3 & -11.2 & -14.2 & 100 & 5.7 & -56 & 1345 & +40 & Rician & Classic $3 \mathrm{~dB}$ \\
\hline 2 & 4 & & -14.2 & 101 & $\mathrm{n} / \mathrm{a}$ & 0 & 70 & $\mathrm{n} / \mathrm{a}$ & Rayleigh & Round \\
\hline 3 & 5 & -19.0 & -19.0 & 200 & $\mathrm{n} / \mathrm{a}$ & -87 & 1358 & $\mathrm{n} / \mathrm{a}$ & Rayleigh & Classic $6 \mathrm{~dB}$ \\
\hline 4 & 6 & -21.9 & -21.9 & 300 & $\mathrm{n} / \mathrm{a}$ & -139 & 1397 & $\mathrm{n} / \mathrm{a}$ & Rayleigh & Classic 3 dB \\
\hline 5 & 7 & -25.3 & -27.9 & 400 & $\mathrm{n} / \mathrm{a}$ & 60 & 1522 & $\mathrm{n} / \mathrm{a}$ & Rayleigh & Classic $6 \mathrm{~dB}$ \\
\hline 5 & 8 & & -30.8 & 401 & $\mathrm{n} / \mathrm{a}$ & -561 & 997 & $\mathrm{n} / \mathrm{a}$ & Rayleigh & Classic $3 \mathrm{~dB}$ \\
\hline 6 & 9 & -24.4 & -24.4 & 500 & $\mathrm{n} / \mathrm{a}$ & 50 & 1529 & $\mathrm{n} / \mathrm{a}$ & Rayleigh & Round \\
\hline 7 & 10 & -28.0 & -28.0 & 600 & $\mathrm{n} / \mathrm{a}$ & 13 & 1572 & $\mathrm{n} / \mathrm{a}$ & Rayleigh & Round \\
\hline 8 & 11 & -26.1 & -31.5 & 700 & $\mathrm{n} / \mathrm{a}$ & -6 & 1562 & $\mathrm{n} / \mathrm{a}$ & Rayleigh & Classic $6 \mathrm{~dB}$ \\
\hline 8 & 12 & & -28.1 & 701 & $\mathrm{n} / \mathrm{a}$ & 4 & 81 & $\mathrm{n} / \mathrm{a}$ & Rayleigh & Round \\
\hline
\end{tabular}

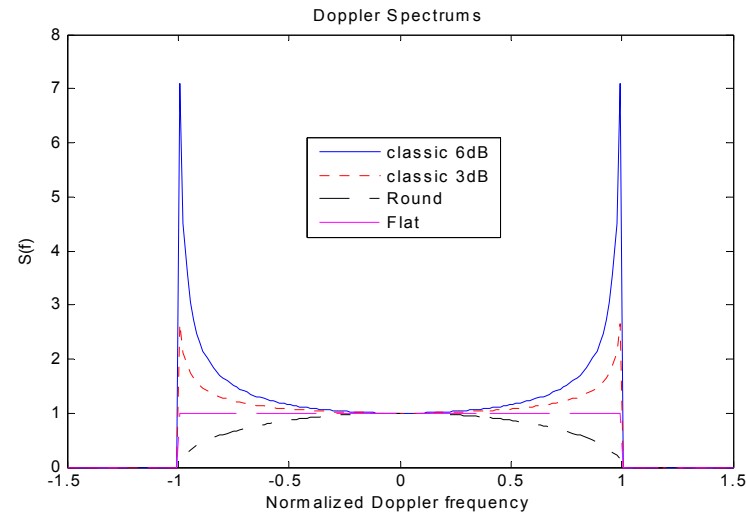

Fig. 4. Doppler spectrum shapes used for channel modeling.

Table IV shows the specific description of this model, which is consistent with vehicle speeds $140 \mathrm{~km} / \mathrm{hr}$. The taps $1,2,5$, and 8 comprise two paths, and the taps 1 and 2 have both Rician and Rayleigh paths. The seventh column "Frequency Shift" and the eighth column "Fading Doppler" denote the center frequency of the fading spectral shape and the half-width of it, respectively. The last column indicates "Fading Spectral Shape" or Doppler spectrum shape. As shown in Fig. 4, four types of fading spectral shapes (classic $6 \mathrm{~dB}$, classic $3 \mathrm{~dB}$, round, and flat) can be used, and three of them are chosen to represent the above model. For the realization of the model, Rayleigh and Rician fading generators are used. They are based on the filtered white Gaussian noise (FWGN) method where real and imaginary WGNs are independently generated and filtered by the Doppler shaping filters. The Doppler shaping filters are made by IFFT-based finite impulse response (FIR) filter design. The Rician fading generator is easily obtained by adding a line-of-sight (LOS) component to the Rayleigh fading generator. The Rayleigh or Rician fading generators shall compose a complete TDL type multipath channel generator. Note that the interpolators are needed to match the sampling time between the multipath channel generator and the WAVE system.

As shown in Fig. 5, the multipath channel generator gives power delay profile (PDP) and Doppler power spectral density (PSD). In Fig. 5(a), the overall $\mathrm{K}$ factor is approximately $4.0 \mathrm{~dB}$ for tap 1 and $-1.8 \mathrm{~dB}$ for tap 2 . Since taps 1 and 2 include the LOS components and their maximum Doppler frequency shifts are less than $100 \mathrm{~Hz}$, they are going to change slowly according to the time. In Fig. 5(b), the Doppler PSD of each path is shown. We obtained similar results to those of the Georgia Tech report. Moreover, we analyzed the multipath channel generator through level crossing rate (LCR) and average fade duration (AFD) which are not presented in this paper for lack of pages.
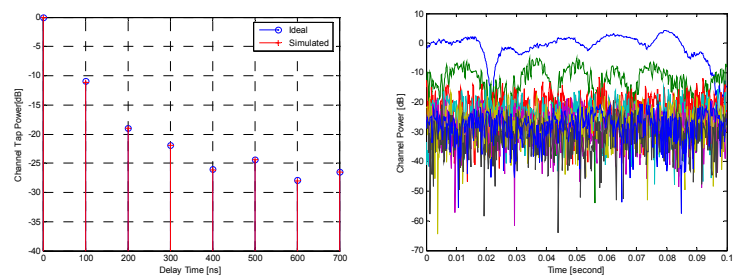

(a) power delay profile and fading waveform
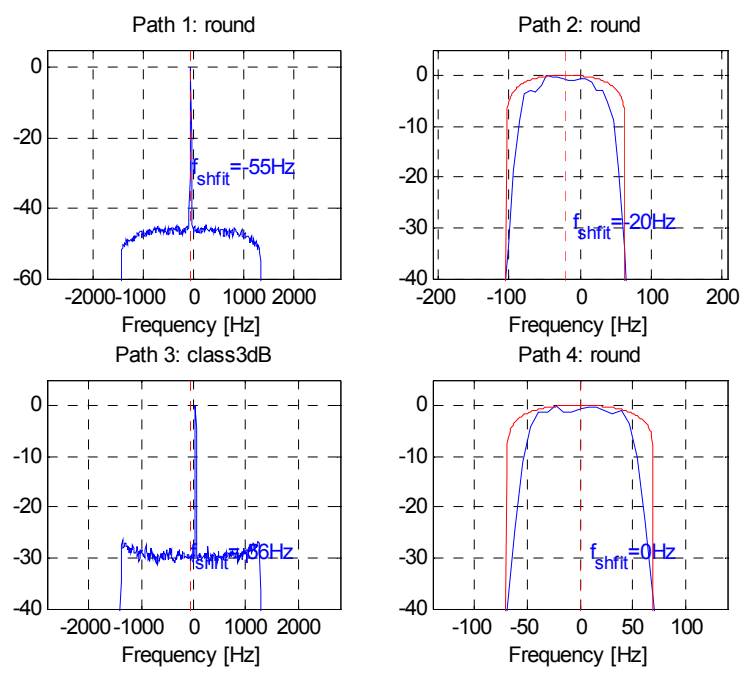

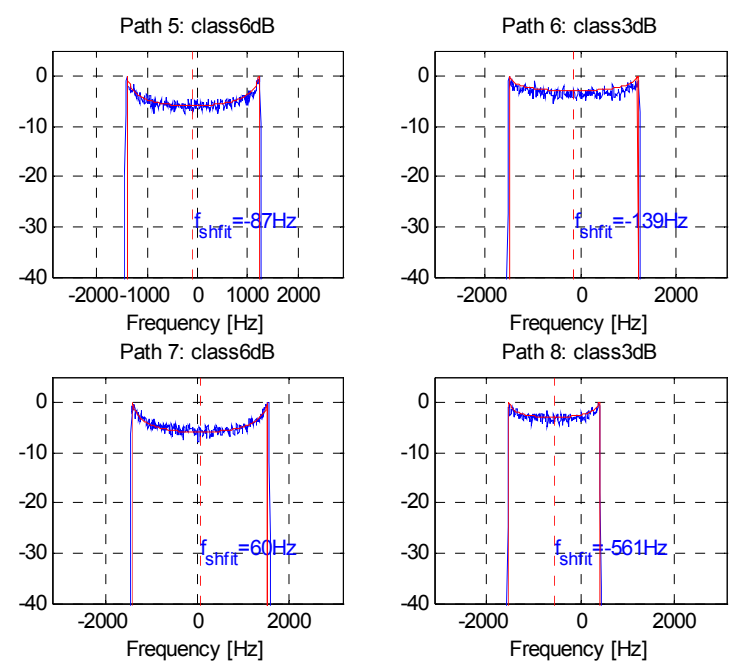

Path 8: class3dB
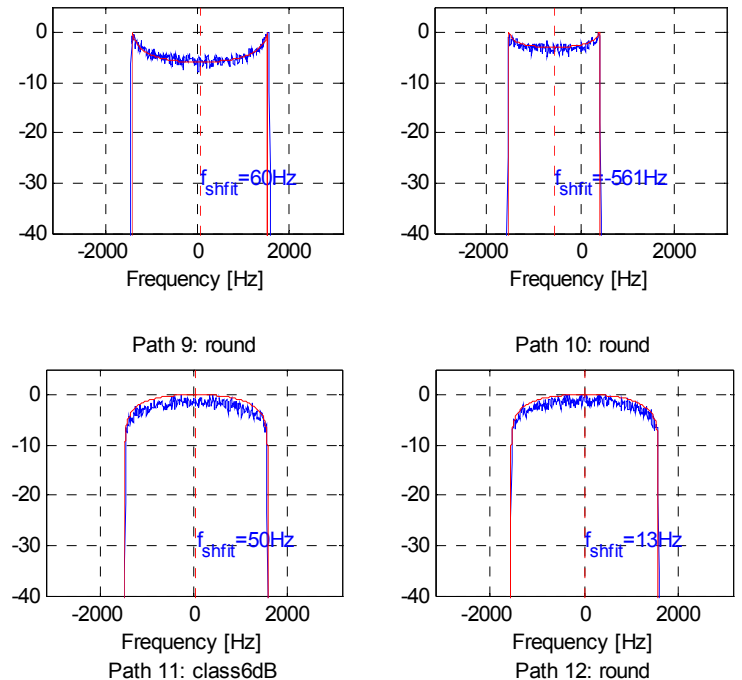

Frequency $[\mathrm{Hz}]$ Path 12: round
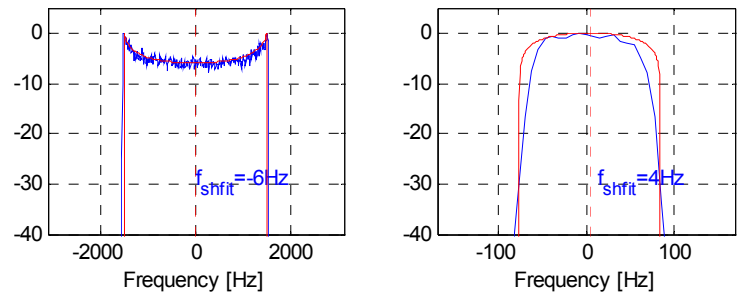

(b) Doppler power spectral densities

Fig. 5. Characteristics of our multipath channel generator.

\section{SIMULATION RESULTS}

In this section, some BER and PER performances of the IEEE $802.11 \mathrm{p}$ WAVE system are presented under small-scale fading models assuming the models are perfectly known to the receiver. Then, effective PSDU sizes are suggested to obtain good performances in accordance with the models when the conventional channel estimation using two long training symbols is used.

In Fig. 6 and Fig. 7, the BER and PER performances are shown according to the transmission modes in Table II. As the worst-case channel, the first model in Table III is considered. The PSDU size is 200 bytes given in the Georgia Tech report. In Fig. 6, the performance of "mode01 " with $1 / 2$ code rate and BPSK modulation is similar to that of "mode-03" with $1 / 2$ code rate and QPSK modulation. The "mode-02" performance looks like "mode-04" performance as well. There is no difference between "mode- 04 " and "mode- 05 " performances. The "mode- 08 " shows the worst performance and requires 20 $\mathrm{E}_{\mathrm{b}} / \mathrm{N}_{0} \mathrm{~dB}$ to get $10^{-3}$ BER performance. In Fig. 7, "mode01 " and "mode- 03 " require about $10 \mathrm{E}_{\mathrm{b}} / \mathrm{N}_{0} \mathrm{~dB}$ to get $10 \%$ PER performance. In case of "mode-02", "mode-04", and "mode- 05 ", about $13 \mathrm{~dB}$ is required. Moreover, "mode06", "mode- 07 ", and "mode- 08 " need about $15 \mathrm{~dB}, 17 \mathrm{~dB}$, and $19 \mathrm{~dB}$, respectively.

In Fig. 8, the PER performance of the maximum data rate mode with $3 / 4$ code rate and 64QAM modulation is shown in the fading models 1,4 , and 10 with PSDU size 200 bytes. In Fig. 9, it is shown in the fading models 6 and 8 with PSDU size 1000 bytes. As the number of the fading model becomes larger, the channel is better. In other words, "WAVE-CH01" denotes the worst channel and "WAVE-CH10" denotes the best channel. Therefore, as shown in Fig. 8, "WAVE-CH01" shows the worst performance whereas "WAVE-CH10" shows the best performance. In order to give $10 \%$ PER performance, "WAVE-CH01" requires about $4 \mathrm{E}_{\mathrm{b}} / \mathrm{N}_{0} \mathrm{~dB}$ more than "WAVE-CH10". In Fig. 9, "WAVE-CH08" shows better performance than "WAVE-Ch06". However, the performance difference between them is about $1 \mathrm{E}_{b} / \mathrm{N}_{0} \mathrm{~dB}$.

In Fig. 10 and Fig. 11, correlation properties of some fading models are shown according to OFDM symbol index. The curve of "WAVE-CH10" looks nearly flat. Its correlation is more than $90 \%$. The curve of "WAVE$\mathrm{CH} 01$ " goes down as OFDM symbol index is increased. It has the worst correlation compared with the others. The correlation property of "WAVE-CH08" is better than "WAVE-CH06". Good correlation property means that the LS channel estimation using two identical long training symbols can work well. Once the channel frequency responses (CFRs) are estimated by the LS channel estimator, they shall be used for the following OFDM symbols without updating themselves. However, as shown in Fig. 12, the LS channel estimation does not provide good PER performance since the difference between the estimated CFR and the real CFR becomes larger. Here, the strongest transmission mode 1 with $1 / 2$ code rate and BPSK modulation is assumed under "WAVE-CH01" and "WAVE-CH10". In "WAVE-CH01" model, the curves of uncoded and coded PER performances are saturated. In "WAVE-CH10" model, they are better than those of "WAVE-CH01" model but they have error floors. In other words, even though "WAVE-CH10" model with the best correlation property is considered, sufficient performance cannot be obtained by the LS channel estimation.

Some channel estimation methods are compared under "WAVE-CH01" model as shown in Fig. 13 where "Perfect" denotes perfect channel estimation, "Conv-LS" denotes the conventional LS channel estimation, and "DD-CE" denotes the decision directed channel estimation without LMMSE smoothing. "Conv-LS" shows the worst performance since it cannot track the 
time variation of "WAVE-CH01". The performance of "DD-CE" is better than "Conv-LS". However, it still suffers from decision errors and noise enhancement. "Proposed" improves "DD-CE" since it can reduce the noise enhancement by LMMSE smoothing. It provides $10^{-1}$ PER performance at $18 \mathrm{E}_{\mathrm{b}} / \mathrm{N}_{0} \mathrm{~dB}$ and has about 4 $\mathrm{E}_{\mathrm{b}} / \mathrm{N}_{0} \mathrm{~dB}$ gain compared with "DD-CE". While the performance difference between "Perfect" and "Proposed" can be reduced by using more sophisticated tracking algorithms, we recommend that the best way is increasing the number of pilot symbols in order to guarantee the fast and accurate communications in emergency situations such as car crashes and natural disasters.

\section{CONCLUSION}

In this paper, the performance of IEEE 802.11p WAVE system was investigated in small-scale fading models which were re-designed for the computer simulations in MATLAB environment. In addition, the IEEE $802.11 \mathrm{p}$ WAVE PHY simulator was developed to provide the BER and PER performances under perfect channel estimation. The problem of the conventional LS channel estimator using two long training symbols was addressed, and an improved channel estimator was proposed where DD-CE and LMMSE smoothing were combined in order to track the time variation of the small-scale fading channel and mitigate noise enhancement. We hope that the simulation results can be used as basic materials to implement the commercialized IEEE 802.11p WAVE system.

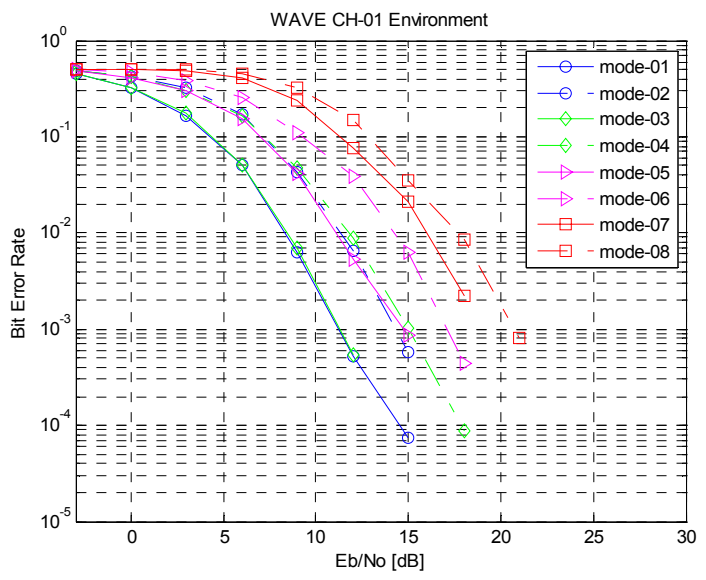

Fig. 6. BER performance according to the transmission modes in the worst fading model : V2V expressway oncoming without wall $300-400 \mathrm{~m}$.

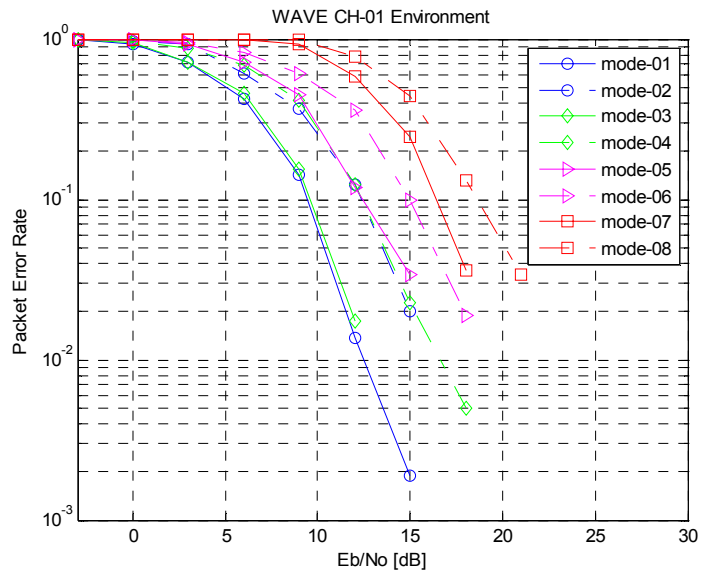

Fig. 7. PER performance according to the transmission modes in the worst fading model : V2V

expressway oncoming without wall $300-400 \mathrm{~m}$.

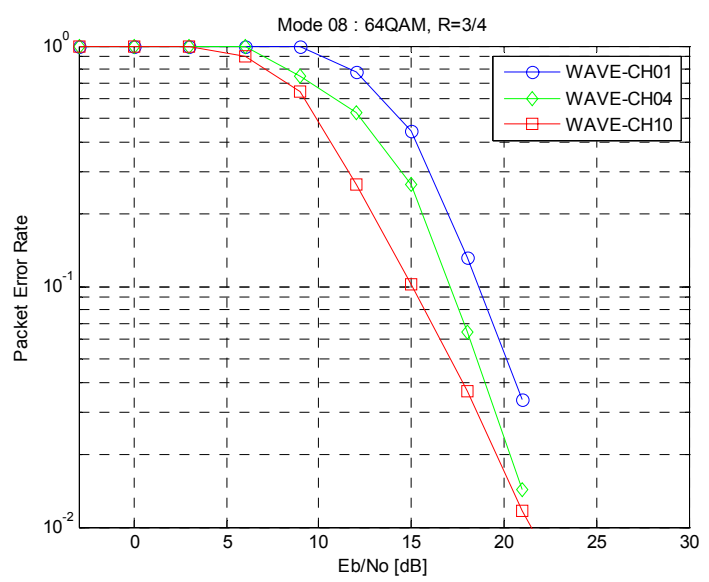

Fig. 8. PER performance of the transmission mode 8 according to some fading models : model 1,4 , and 10 with PSDU size 200 bytes.

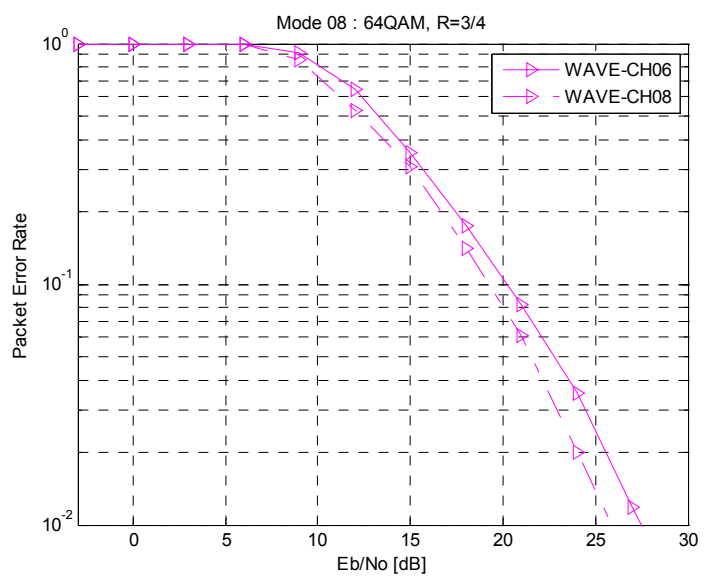

Fig. 9. PER performance of the transmission mode 8 according to some fading models : model 6 and 8 with PSDU size 1000 bytes. 


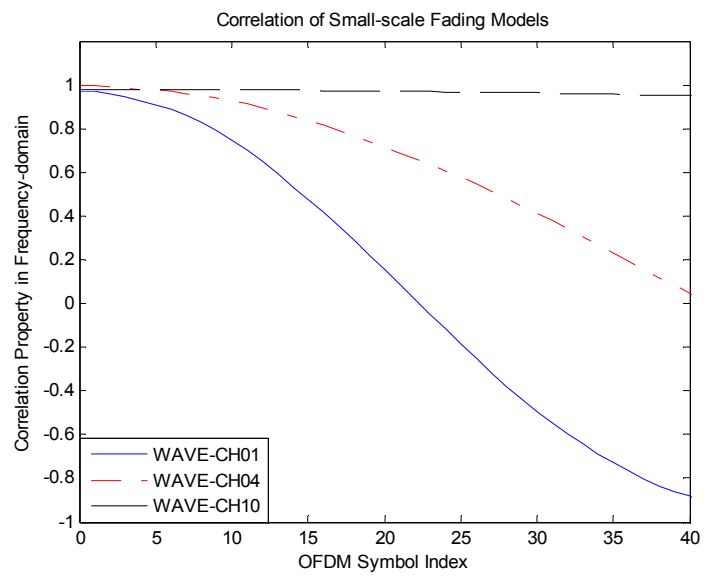

Fig. 10. Correlation properties of some fading models according to OFDM symbol index : models 1,4 , and 10

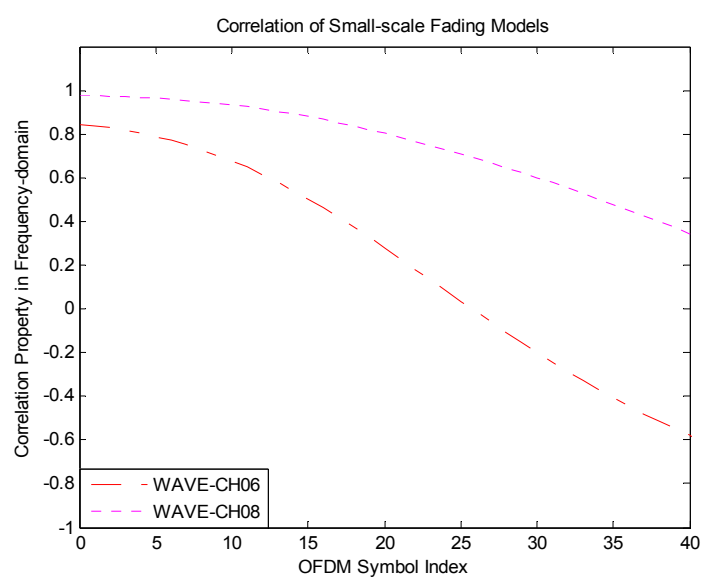

Fig. 11. Correlation properties of some fading models according to OFDM symbol index : models 6 and 8 .

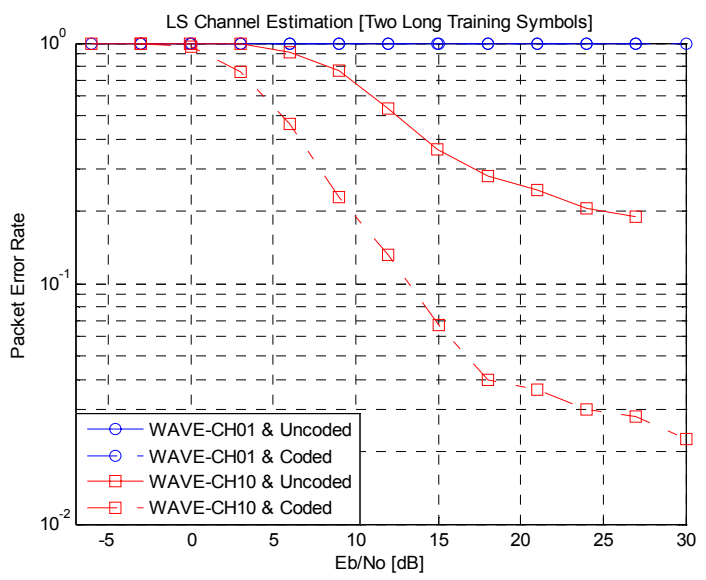

Fig. 12. Uncoded and coded PER performance of the transmission mode 1, assuming the LS channel estimation using two long training symbols.

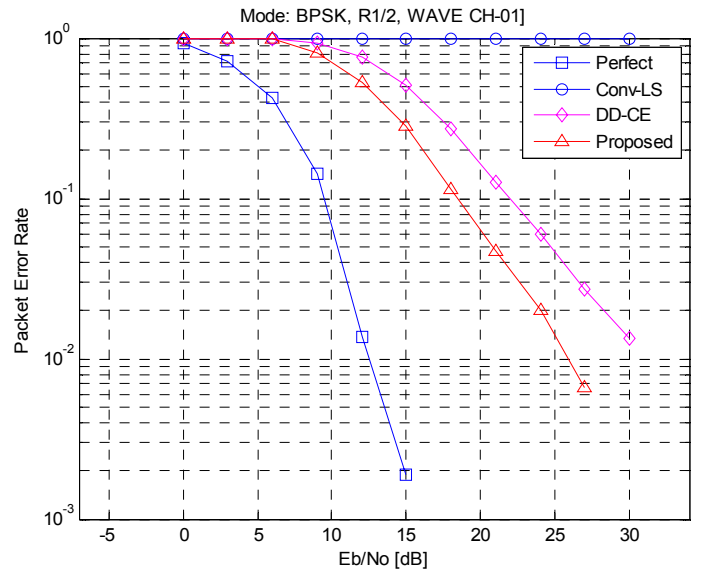

Fig. 13. Coded PER performance of the transmission mode 1 with the proposed channel estimator.

\section{REFERENCES}

[1] IEEE P802.11p/D1.0, Draft Amendment to Standard for Information Technology-Telecommunications and information exchange between systems-Local and Metropolitan networksspecific requirements-Part 11: 'Wireless LAN Medium Access Control(MAC) and Physical Layer(PHY) specifications, Amendment 3: Wireless Access in Vehicular Environment', Feb. 2006.

[2] Jiang, D., and Delgrossi, L. : 'IEEE 802.11p: Towards an international standard for wireless access in vehicular environments', VTC-2008 Spring, May 2008, pp. 2036-2040.

[3] S. Eichler : 'Performance evaluation of the IEEE 802.11p WAVE Communication Standard', VTC-2007 Fall, Sept. 2007, pp. 2199. 2203.

[4] IEEE P.802.11 Wireless LANs : 'WAVE Channel Model', Georgia Institute of Technology, Nov. 2006.

[5] Acosta-Marum, G., and Ingram, M. A. : 'Six time- and frequencyselective empirical channel models for vehicular wireless LANs', IEEE Vehicular Technology Magazine, Dec. 2007, vol. 2, pp. 4-11.

[6] Abdulhamid, H., Abdel-Raheem, E., and Tepe , K. E. : 'Channel estimation for $5.9 \mathrm{GHz}$ dedicated short-range communications receiver in wireless access vehicular environments', IET Communications, Dec. 2007, vol. 1, pp. 1274-1279.

[7] Terry, J., and Heiskala, J. : 'OFDM Wireless LANs: A Theoretical and Practical Guide' (SAMS, 2002).

[8] Edfors, O., Sandel, M., van de Beek , J.-J., Wilson, S. K., and Börjesson, P. O. : 'OFDM channel estimation by singular value decomposition', IEEE Trans. Comm., July 1998, vol. 46, no. 7, pp. 931-939. 


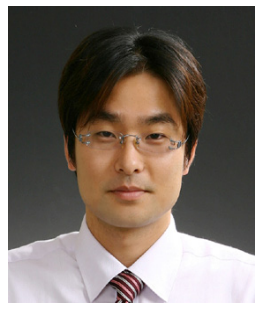

Jeongwook Seo received the B.S. and M.S. degrees in the Department of Telecommunication \& Information Engineering from Korea Aerospace University, Gyeonggi, Korea, in 1999 and 2001, respectively. He is currently working toward the Ph.D. degree in the School of Electrical \& Electronic Engineering from Yonsei University, Seoul, Korea. Since 2001, he has been with Advanced Mobile Technology Research Center in Korea Electronics Technology Institute (KETI), Gyeonggi, Korea. His research interests include statistical signal processing, digital communications, and OFDM-based wireless systems.

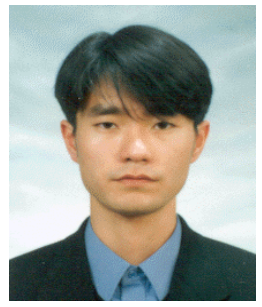

Jae-Min Kwak received the B.S., M.S., and Ph.D. degrees in the Department of Telecommunication \& Information Engineering from Korea Aerospace University, Gyeonggi, Korea, in 1998, 1999, and 2002, respectively.

He was a Managerial Researcher with the SoC Research Center in Korea Electronics Technology Institute (KETI) by Feb. 2008, and he has been a assistant professor in the Devision of Marine Electronics \& Communication Engineering, Mokpo National Maritime University, since Mar. 2008. His research interests include digital communication signal processing, OFDM and UWB based wireless communication systems.

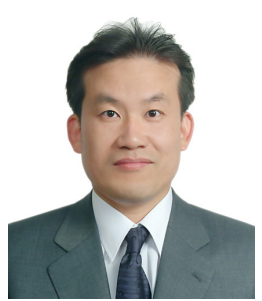

Dong Ku Kim received the B. Eng. Degree from Korea Aerospace University, Korea, in 1983 and the M.Eng and Ph.D. degrees from the University of Southern California, Los Angeles, in 1985 and 1992, respectively. He was a Research Engineer with the Cellular Infrastructure Group, Motorola by 1994, and he has been a professor in the school of Electrical and Electronic Engineering, Yonsei University, Seoul, since 1994. He is a director of Radio Communication Research Center at Yonsei University. He has been also a director of Qualcomm Yonsei CDMA Joint Research Lab since 1999. His main research interests are multi-user mulit-mode MIMO, OFDM, scheduling schemes, mobile multihop relay, and location finding algorithms. 